\title{
Enhanced Version of Multi-algorithm Genetically Adaptive for Multiobjective optimization
}

\author{
Wali Khan Mashwani \\ Department of Mathematics, \\ Kohat University of Science \& Technology, \\ Khyber Pakhtunkhwa (KPK), Pakistan \\ Abdellah Salhi \\ Department of Mathematical Sciences, \\ University of Essex, \\ Wivenhoe Park,Colchester, UK
}

\author{
Muhammad Asif jan \\ Department of Mathematics, \\ Kohat University of Science \& Technology, \\ Khyber Pakhtunkhwa (KPK), Pakistan
}

\author{
Rashida Adeeb Khanum \\ Department of Mathematics, \\ Jinnah College for Women Peshawar, \\ Khyber Pakhtunkhwa (KPK), Pakistan
}

\author{
Muhammad Sulaiman \\ Department of Mathematics, \\ Abdul Wali Khan University, Mardan, \\ Khyber Pakhtunkhwa (KPK), Pakistan
}

\begin{abstract}
Multi-objective EAs (MOEAs) are well established population-based techniques for solving various search and optimization problems. MOEAs employ different evolutionary operators to evolve populations of solutions for approximating the set of optimal solutions of the problem at hand in a single simulation run. Different evolutionary operators suite different problems. The use of multiple operators with a selfadaptive capability can further improve the performance of existing MOEAs. This paper suggests an enhanced version of a genetically adaptive multi-algorithm for multi-objective (AMALGAM) optimisation which includes differential evolution (DE), particle swarm optimization (PSO), simulated binary crossover (SBX), Pareto archive evolution strategy (PAES) and simplex crossover (SPX) for population evolution during the course of optimization. We examine the performance of this enhanced version of AMALGAM experimentally over two different test suites, the ZDT test problems and the test instances designed recently for the special session on MOEA's competition at the Congress of Evolutionary Computing of 2009 (CEC'09). The suggested algorithm has found better approximate solutions on most test problems in terms of inverted generational distance (IGD) as the metric indicator.
\end{abstract}

Keywords-Multi-objective optimization, Multi-objective Evolutionary algorithms (MOEAs), Pareto Optimality, Multi-objective Memetic Algorithm (MOMAs).

\section{INTRODUCTION}

Multi-objective evolutionary optimization is a subject of intense interest in all fields of Science, Engineering, Economics, Logistics and others. Multi-objective optimization problems (MOPs) have more than one conflicting objective function and they have many real-world applications [6], [59]. A general MOP can mathematically be formulated as follows.

$$
\begin{aligned}
& \text { minimize } F(x)=\left(f_{1}(x), \ldots, f_{m}(x)\right)^{T} \\
& \text { subject to } x \in \Omega
\end{aligned}
$$

where $\Omega$ is the decision variable space, $x=\left(x_{1}, x_{2}, \ldots, x_{n}\right)^{T}$ is an individual or solution and $x_{i}, i=1, \ldots, n$ are their decision variables, $F(x): \Omega \rightarrow R^{m}$ consists of $m$ real valued objective functions and $R^{m}$ is called the objective space. If $\Omega$ is a closed and connected region in $R^{n}$ and all the objective functions in (1) are continuous for $x$, we call it a continuous MOP. Furthermore, if $m \geq 3$, then problem (1) is said to be a many objectives problem. In single objective optimization, the main focus is on the decision space while in multi-objective optimization, the focus is mainly on the objective space because objective values are used in checking for optimality [43]. In practical applications of optimization, it is very common that the objective functions of the MOP conflict with one another or are mostly incommensurable. One needs a set of optimal solutions to solve these problems.A solution $u=\left(u_{1}, u_{2}, \ldots, u_{n}\right) \in \Omega$ is said to be Pareto optimal if there exist no another solution $v=\left(v_{1}, v_{2}, \ldots, v_{n}\right) \in \Omega$ such that $f_{j}(u) \leq f_{j}(v)$ for all $j=1, \ldots, m$ and also $f_{j}(u)<f_{j}(v)$ for at least index $k$. An objective vector is said to be Pareto optimal if their corresponding decision vector is Pareto optimal. All Pareto optimal solutions in the decision space of MOP is called Pareto set (PS) and their corresponding image in their objective space is called Pareto front (PF). The idea Pareto optimality was first proposed by Francis Ysidro Edgeworth in 1881 and then later on generalized by Vilfredo Pareto in 1986 as discussed in [12], [10].

MOEAs are highly effective and powerful stochastic techniques which can find a set optimal solutions in a single simulation run due to their population-based nature, unlike traditional mathematical programming.In the past two decades, and since the inception of vector evaluated GA (VEGA) [48], different types of MOEAs have been suggested, the Pareto dominance based MOEAs [11], [13], [61], [60], [44], [19], [18], [9], [27]), the decomposition based MOEAs [21], [20], [54], [8], [7], [30], [56], [58], [55], [1], [34], [32], [39], [42], [41], [26], [25], [35], [37], [33], [41]), and Indicator Based algorithms [63], [5], [3], [22], [4], [2], [14]. They mainly emphasize three conflicting goals: firstly, the final approximate Pareto front $(\mathrm{PF})$ should be as close as possible to the true PF; secondly, the final set of Pareto optimal solutions should be uniformly distributed and diverse over the true PF of the problem (1); thirdly, the approximated PF should capture the whole spectrum of the true PF. Different fitness assignment procedures, elitism and diversity promoting strategies are found in the current literature of evolutionary computing (EC). 
The Pareto dominance concept of MOEAs is very common for solving MOPs [28], [12]. To promote diversity, most of these algorithms use different diversity techniques such as fitness sharing, niching, the kernel approach, the nearest neighbour approach, the histogram technique, crowding or clustering, a relaxed form of dominance and restricted mating [11]. Among them, a fast non-dominated sorting algorithm (NSGAII) [13], SPEA2 or improving the strength Pareto evolutionary algorithm [60], the Pareto archive evolution strategy (PAES) [27], multi-objective genetic algorithm (MOGA) [18], and niched Pareto genetic algorithm (NPGA) [19] are long-familiar and well known approaches. They have shown good behaviors in several comparative analysis.

Multiobjective evolutionary algorithms (MOEAs) are extremely useful for dealing with MOPs. They evolve their population solutions and provide Pareto optimal solutions in single simulation unlike traditional optimization techniques. In the past two decades, since the inception of vector evaluated genetic algorithm (VEGA) [48], several MOEAs have been suggested and [13], [61], [60], [17], [44], [19], [9], [11], [49], [59], [32], [36], [38]. they have successfully tackled various types of MOPs [16], [25], [35], [37], [33]. In general, classical MOEAs can be divided into three main different classes, namely, the Pareto dominance based MOEAs (e.g., [11], [13], [61], [60], [44], [19], [18], [9], [27]), the decomposition based MOEAs (e.g., [21], [20], [54], [8], [7], [30], [56], [58], [55], [1], [34], [32], [39], [42], [41], [26]), and Indicator Based algorithms (e.g., [63], [5], [3], [22], [4], [2], [14]). All these algorithms try to obtain a set of Pareto optimal solutions with three main features, firstly, It should close as much as possible to the true PF. Secondly, the approximated set should require expand uniformly distributive all over the true PF of the problem (1). Thirdly, final Parerto optimal solutions obtained by particular MOEA should require to desirably capture the whole spectrum of the PF of the problems. The existing algorithms implement different fitness assignment procedures to to evolve their population in order to achieve aforementioned three goals subject to No free Lunch concept [29]. The Pareto dominance concept based MOEAs thoroughly applied for coping with MOPs [28], [12]. To promote diversity, most of these algorithms are utilizing different diversity techniques such as fitness sharing, niching approach, Kernel approach, nearest neighbour approach, histogram technique, crowding or clustering, relaxed form of dominance and restricted mating [11]. Among them, a fast non-dominated sorting algorithm (NSGA-II) [13], SPEA2 or improving the strength Pareto evolutionary algorithm [60], the Pareto archive evolution strategy (PAES) [27], multi-objective genetic algorithm (MOGA) [18], and niched Pareto genetic algorithm (NPGA) [19] have been chosen in several comparative analysis.

Multi-objective memetic algorithms (MOMAs) form a new and attractive area of research in EC. They are inspired by models of adaptation found in nature. They are known as Baldwinian EAs, Lamarckian EAs, cultural algorithms, or genetic local search and hybrid MOEAs [45]. Hybrid MOEAs have been developed with the aim to overcome the shortcomings of stand-alone MOEAs [34], [32], [39], [42], [40].

A genetically adaptive multi-algorithm for multi-objective (AMALGAM) optimisation is recently developed for solving both multi-objective optimization problems [52] and single optimization problems [53]. It employs multiple search operators for its population evolution. The search operators used include the particle swarm optimizer (PSO) [15], differential evolution (DE) [47] and NSGA-II [13] and allocates resources dynamically to each search operators based on their individual performances. It does not involve any decomposition as in MOEA/D (multio-bjective evolutionary algorithm based on decomposition) [54].

MOEA/D [54] decomposes the approximated PF of the given MOP into a number of different single objective optimization subproblems (SOPs). It then optimizes all SOPs simultaneously using generic evolutionary algorithm. MOEA/D paradigm have tackled diverse benchmark functions and it has several enhanced versions [31], [34], [32], [39], [36], [42]. In this paper, our main objective is to further improve the algorithmic performance of ALMAGAM by employing by employing multiple search operators including the differential evolution (DE) [46], particle swarm optimization (PSO) [15], simulated binary crossover (SBX) [24], Pareto archive evolution strategy (PAES) [23] and simplex crossover (SPX) [50] with self-adaptive alternative procedures for dealing with both CEC'09 test instances [57] and ZDT test problems [62].

The main objective in this paper to develop an enhanced version of ALMAGAM by employing multiple search operators such as differential evolution (DE) [46], particle swarm optimization (PSO) [15], simulated binary crossover (SBX) [24], Pareto archive evolution strategy (PAES) [23] and simplex crossover (SPX) [50] with self-adaptive procedures for dealing with both CEC'09 test instances [57] and ZDT test problems [62].

The rest of this paper is organized as follows. Section II outlines the framework of the enhanced version of the genetically adaptive multi-algorithm multi-objective (AMALGAM)method. Section III presents experimental results obtained with the enhanced AMALGAM on both CEC'09 [57] and five ZDT test problems [62]. Section IV is devoted to a discussion on experimental results. Section V finally concludes this paper and suggest further areas of research on this topic an related ones.

\section{ENHANCED VERSION OF MULTI-ALGORITHM Genetically Adaptive for Multiobjective OPTIMIZATION}

Algorithm 1 outlines the framework of the an enhanced version of the AMALGAM. In Sept 1, a population $P$ with size $N$ has been generated uniformly and randomly within the search space of the given MOPs. We then evaluate the fitness values of solution of population $P$. We calculate the crowding distance of each member of population after categorize them into different layers by using fast non-dominating sorting procedure adopted in NSGA-II [13] framework. After this, an Algorithm 1 divide the whole population according to $k$ number of search operators in order to work each search operator on specified number of sub-populations $N_{1}, N_{2}, N_{3}$ to generate $Q$ offspring population of sizes whose sum is equal to $N$. We have used five different search operators such as differential evolution (DE) [46], particle swarm optimization (PSO) [15], simulated binary crossover (SBX) [24] and Pareto archive evolution strategy (PAES) [23] and simplex crossover (SPX) [50] in 
the evolutionary process of the suggested algorithm. Each individual search operator is getting resources at population level according to their current individual performance based on self-adaptive procedure as explained in subsection II-A.

$\overline{\text { Algorithm } 1 \text { Enhanced Version of Multi-algorithm Genetically }}$ Adaptive for Multiobjective optimization

1: Input:

2: MOP: the multiobjective optimization problem; $N$ : the population size and other main parameters; $\mathbf{F}_{\text {eval }}$ : maximum function evaluations;

3: Output: $\left\{x^{1}, \ldots, x^{N}\right\}$ and $\left\{F\left(x^{1}\right), \ldots, F\left(x^{N}\right)\right\}$;

4: Generate an initial population $P$ of size $N$ uniformly and randomly.

5: Calculate the F-function values of each member of the $P$ population.

6: Assign rank to each member of $P$ using fast nondominating procedure.

7: Assign sub-populations $P=\left\{P_{1}, P_{2}, \ldots, P_{k}\right\}$ to $k$ operators for creating an offspring population $Q=$ $\left\{Q_{1}, Q_{2}, \ldots, Q_{k}\right\}$ of size N.

8: Calculate F-function values of $Q$ offspring population.

9: Assign rank to each member of $Q$ using fast nondominating procedure.

10: Combine the new and old population $P$ and $Q, R=P \cup Q$.

11: Select population $P$ of size $N$ from population $R$ of size $2 N$ based on their ranks and crowding distances for next generation.

12: Update $N$ best individuals among $C$ population with high ranks and crowding density.

13: Update $P=\left\{P_{1}, P_{2}, \ldots, P_{k}\right\}$ (Explanation can be found in subsection II-A) based on the individual performances of each search operator.

\section{A. Alternative Adaptive Resources Allocation Scheme}

- We calculate the number of solutions that successfully enter to the next generation in the evolutionary process of enhanced version of AMALGAM. A successful solution is rewarded by 1 and unsuccessful by 0 . An efficient operator gets more resources in the form of subpopulation to be operate on them as compared to weaker one.

- Let $\delta_{k}, k=1,2, \ldots, q$ are total number of nondominated solutions produced by $q$ search operators (i.e, differential evolution (DE) [46], particle swarm optimization (PSO) [15], simulated binary crossover (SBX) [24], Pareto archive evolution strategy (PAES) [23] and simplex crossover (SPX) [50]) that enter successfully to next generation are convert into normalized form to develop probability formula (3)

$$
\begin{gathered}
\mathbf{P}_{\mathbf{k}}=\frac{\zeta_{\mathbf{k}}}{\sum_{\mathbf{k}=\mathbf{1}}^{\mathbf{q}} \zeta_{\mathbf{k}}}, \text { where } \quad \zeta_{k}=\frac{\delta_{k}}{\sum_{k=1}^{q} \delta_{k}} \\
P_{k}=\alpha P_{k-1} \times N+(1-\alpha) P_{k} \times N
\end{gathered}
$$

Where $P_{k}$ is the current and $P_{k-1}$ is the previous probability of successes of the $k$ search operators. More importantly, the above mentioned dynamic resources allocation did not switch on at every generation of proposed algorithm.It can allocate resources at every multiple of $5^{\text {th }}$ generation to tackle ZDT test problems [62]. The suggested enhanced AMALGAM allocates resources to each of its embedded search operator at every multiple of $10^{t h}$ generation for dealing with CEC'09 test instances [57].

\section{PARAMETERS SETTING AND EXPERIMENTAL RESULTS}

We have carried out Experiments using benchmark functions with two and three objectives. The ZDT test [62] were tackled with parameter settings as explained in the subsection III-A while CEC'09 [57] were handled with parameter settings are explained in the subsection III-B, respectively.

\section{A. Parameter Settings for ZDT Problems}

- $\quad N=100$ : population size for 2-objective test instances.

- $F=0.5$ : scaling factor of the DE;

- $C R=0.5$ : crossover probability for $\mathrm{DE}$;

- $\quad w$ is the inertia factor which lies in $[0.8,1.2]$;

- $\quad c_{1}$ and $c_{2}$ are the two acceleration constant or acceleration coefficients that usually lies between 1 and 4;

- $u_{r} \in[-1,1]$ is a continuous uniform random number

- $w=0.5+\operatorname{rand} / 2$ : inertia factor which lies in $[0.8,1.2]$ and $\xi=1$;

- $c_{1}=c_{2}=1.5$ : acceleration constant or acceleration coefficients that usually lies between 1 and 4;

- $\quad F_{\text {eval }}=25000$ : maximum function evaluations;

\section{B. Parameter Settings for CEC'09 Test Instances}

This subsection explains the parameters setting to validate enhanced AMALGAM on CEC'09 test instances [57].

- $\quad N=600$ : population size for 2-objective test instances;

- $\quad N=1000$ : for 3-objective test instances;

- $F=0.5$ : scaling factor of the DE;

- $C R=1$ : crossover probability for $\mathrm{DE}$;

- $\quad F_{\text {eval }}=300,000$ : maximum function evaluations;

\section{Performance Indicators}

Two main goals for dealing with multiobjective evolutionary optimization are very important: 1) convergence towards the Pareto-optimal front, 2) to find uniform and welldistributive set of multiple solutions that cover the whole true PF of the problem at hand [12]. Several performance metrics are found in the specialized literature of evolutionary computing (EC) [51], [13], [12], [64] which are using to judge which algorithm is better than others and in what aspects. Inverted generational distance (IGD) [64], [57], relative hypervolume [51], [12], Gamma $\Upsilon$ and delta $\Delta$ [12], [13] which are commonly in several comparative analysis of different algorithms. The aforementioned performance indicators can 
use only if the reference set for the test problems are known in advance or available. In this paper, we have used the Inverted Generational Distance (IGD) as performance indicator to judge the quality of final approximated set of Pareto optimal solutions obtained by proposed algorithm in comparison with other MOEAs.

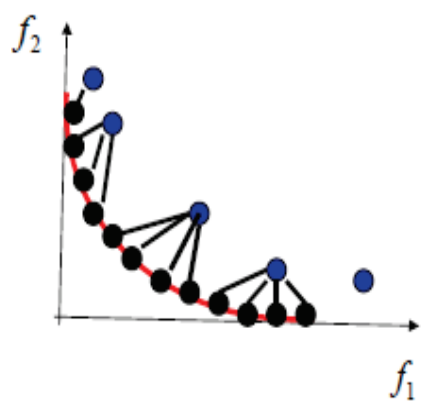

Fig. 1. Inverted Generation Distance (IGD) has been used as a Performance Indicator. The above solutions (blue fill circle) are approximated solution of the algorithm and (black fill circle) are the Pareto solutions uniformly distributive along the Pareto Front (PF).

Let $P^{*}$ be a set of uniformly distributed points along the PF. Let $A$ be an approximate set to the PF, the average distance from $P^{*}$ to $A$ is defined as [57]:

$$
D(A, P)=\frac{\sum_{v \in P^{*}} d(v, A)}{\left|P^{*}\right|}
$$

where $d(v, A)$ is the minimum Euclidean distance between $v$ and the points in $A$. If $P^{*}$ is large enough to represent the PF very well, $D(A, P)$ could measure both the diversity and convergence of $A$ in a sense. The closer the IGD metric values, better is the approximation set. We have used $P^{*}=500$ in our carried experiments to tackle 2-objectives test instances and $P^{*}=1000$ to solve 3-objectives problems. For two objectives case, the IGD metric is pictorially depicted in the figure 1. It measures both the convergence and the spread of the obtained solutions. Smaller IGD-metric values, better will be approximated set of Pareto optimal of solutions of corresponding MOEAs.

\section{DISCUSSION OF THE EXPERIMENTAL RESUlTS OBTAINED ON ZDT AND CEC'09 TEST INSTANCES}

The simulation related parameters are as follows.

- Operating system: Windows XP Professional

- Programming language of the algorithms: Matlab

- $\quad$ CPU: Core 2 Quad $2.4 \mathrm{GHz}$

- $\mathrm{RAM}$ : 4 GB DDR2 $1066 \mathrm{MHz}$

- 30 independent runs were performed on each test problem.

\section{A. Discussion of IGD-metric Values}

Table I and Table II record the IGD-metric values in terms of minimum (Best), Median, Mean, and standard deviation(std) and maximum (worst) which are found by enhanced AMALGAM and NSGA-II [13], respectively. These statistics have been collected by executing each algorithm 30 times independently with different random seeds on each ZDT test problem [62] and CEC'09 test instance [57]. The average CPU time spent by each algorithm are also provided in the last columns of I and Table II. It is evident from these Tables, that enhanced AMALGAM has found better approximated solution set with reduced the IGD-metric values as compared to NSGAII [13] for most test problems. In most test problems, global convergence has been got for both test suites of problems. However, the complete Pareto front for some CEC'09 test instances have not been attained by enhanced AMALGAM in multiobjective optimization context. The primary reasons of this weak performance could be reason of the complicated objective functions profile of some CEC'09 test instances [57] which are mostly multi-modal near the global Pareto-optimal frontier and a slight perturbation in their optimization variables causes their solutions to become dominated.

\section{B. Discussion of the Pareto Fronts of ZDT and CEC'09 Test Instances.}

Table I and Table II record the IGD-metric values in terms of minimum (Best), Median, Mean, and standard deviation (std) and maximum (worst) which are found by enhanced AMALGAM and NSGA-II [13], respectively. These statistics have been collected by executing each algorithm 30 times independently with different random seeds on each ZDT test problem [62] and CEC'09 test instance [57]. The average CPU time spent by each algorithm are also provided in the last columns of I and Table II. It is evident from statistics gathered in these Tables, that enhanced AMALGAM has found a better approximate solution set with reduced IGDmetric values compared to those of NSGA-II [13] for most test problems. In most test problems, global convergence has been achieved for both test suites of problems. However, the complete Pareto front for some CEC'09 test instances has not been attained by the enhanced AMALGAM algorithm, in the multi-objective optimization context. The primary reasons for this weak performance could be the complicated objective functions profile in some CEC'09 test instances [57]; these are mostly multi-modal near the global Pareto-optimal frontier and a slight perturbation in their optimization variables causes their solutions to become dominated.

Figure 2 and Figure 6 depict the approximated Pareto front (PF) against the real PF of ZDT test problems displayed by enhanced AMALGAM and NSGA-II [13], respectively. These figures indicate that both algorithms have found better approximated PF in their best run among 30 independent runs on each ZDT test problem. We have plotted 30 PFs together in Figures 3 and Figures 7 of the enhanced AMALGAM and NSGA-II [13], respectively. These figures indicate that enhanced AMALGAM has displayed all 30 in better distribution ranges in all 30 independent runs as compared to NSGA-II [13].

Figures 4 display the best approximated PF of the CEC'09 test instances as demonstrated by enhanced AMALGAM 
TABLE I. The IGD-METRIC VALUES OF THE ENHANCED AMALGAM FOR ZDT1-ZDT4 AND ZDT6. AVG-T MEANS AVERAGE CPU TIME IN SECONDS.

\begin{tabular}{|c|c|c|c|c|c|c|}
\hline \hline ZDT & Best & Median & Mean & St.Dev. & worst & AVG-T \\
\hline ZDT1 & 0.004301 & 0.004521 & 0.004603 & 0.000223 & 0.005065 & 14.940230 \\
\hline ZDT2 & 0.004235 & 0.004794 & 0.004613 & 0.000258 & 0.005643 & 14.633287 \\
\hline ZDT3 & 0.005067 & 0.005498 & 0.005565 & 0.000170 & 0.006134 & 14.580179 \\
\hline ZDT4 & 0.004696 & 0.005175 & 0.005235 & 0.000162 & 0.005575 & 14.696021 \\
\hline ZDT6 & 0.003615 & 0.004037 & 0.004045 & 0.000175 & 0.004691 & 14.395623 \\
\hline
\end{tabular}

TABLE II. The IGD-MEtric VALUes of the NSGA-II [13] FOR DEALING WITH ZDT1-ZDT4 AND ZDT6. AVG-T MEANS AVERAGE CPU TIME IN SECONDS.

\begin{tabular}{|c|c|c|c|c|c|c|}
\hline \hline ZDT & Best & Median & Mean & St.Dev. & worst & AVG-T \\
\hline ZDT1 & 0.0042193 & 0.004472 & 0.004369 & 0.000139 & 0.004258 & 18.01 \\
\hline ZDT2 & 0.0043213 & 0.004649 & 0.004656 & 0.000182 & 0.005011 & 22.85 \\
\hline ZDT3 & 0.005132 & 0.00546 & 0.00912 & 0.01388 & 0.0602182042 & 17.596 \\
\hline ZDT4 & 0.00482 & 0.006421 & 0.00825 & 0.009649 & 0.059017370 & 22.85 \\
\hline ZDT6 & 0.005606 & 0.007045 & 0.007003 & 0.0005878 & 0.0080474634 & 19.90 \\
\hline
\end{tabular}

TABLE III. The IGD-METRIC VALUES OBTAINED By ENHANCED AMALGAM OVER CEC'09 TEST INSTANCES. AVG-T MEANS AVERAGE CPU TIME IN SECONDS.

\begin{tabular}{|c|c|c|c|c|c|c|}
\hline \hline CEC'09 & best & mean & median & st. dev. & worst & AvG-T \\
\hline UF1 & 0.028431 & 0.058596 & 0.057886 & 0.008465 & 0.070089 & 286.307532 \\
\hline UF2 & 0.011235 & 0.018219 & 0.013157 & 0.001349 & 0.016859 & 288.558110 \\
\hline UF 3 & 0.091864 & 0.134375 & 0.136495 & 0.022836 & 0.198629 & 297.999586 \\
\hline UF 4 & 0.040348 & 0.041052 & 0.041039 & 0.000337 & 0.041667 & 286.159044 \\
\hline UF5 & 0.165346 & 0.171338 & 0.171421 & 0.002796 & 0.176307 & 258.823135 \\
\hline UF 6 & 0.068597 & 0.079037 & 0.078632 & 0.005978 & 0.088905 & 308.462841 \\
\hline UF7 & 0.014935 & 0.017689 & 0.017787 & 0.001267 & 0.020866 & 290.402351 \\
\hline UF8 & 0.103734 & 0.234131 & 0.230672 & 0.026091 & 0.261546 & 720.849149 \\
\hline UF 9 & 0.056715 & 0.067789 & 0.114643 & 0.085653 & 0.325885 & 700.875474 \\
\hline UF10 & 0.273393 & 0.327878 & 0.326937 & 0.020029 & 0.360963 & 686.032888 \\
\hline
\end{tabular}

TABLE IV. THE IGD-METRIC VALUES GENERATE By NSGA-II [13] IN 30 INDEPENDENT RUNS FOR CEC'09 TEST INSTANCES. AVG-T MEANS AVERAGE CPU TIME IN SECONDS.

\begin{tabular}{|c|c|c|c|c|c|c|}
\hline \hline CEC'09 & best & mean & median & st. dev. & worst & AvG-T \\
\hline UF1 & 0.051996 & 0.106873 & 0.096076 & 0.024862 & 0.128739 & 759.27 \\
\hline UF2 & 0.016012 & 0.019849 & 0.020050 & 0.001407 & 0.023589 & 518.07 \\
\hline UF3 & 0.066353 & 0.098234 & 0.097065 & 0.017958 & 0.134235 & 491.95 \\
\hline UF4 & 0.052199 & 0.054388 & 0.054551 & 0.001274 & 0.056679 & 393.60 \\
\hline UF5 & 1.523087 & 1.671735 & 1.676288 & 0.099452 & 1.844279 & 792.28 \\
\hline UF6 & 0.705834 & 0.762023 & 0.762271 & 0.028052 & 0.831784 & 822.79 \\
\hline UF7 & 0.067270 & 0.114403 & 0.112305 & 0.012055 & 0.125719 & 722.11 \\
\hline UF8 & 0.095436 & 0.108548 & 0.120433 & 0.030475 & 0.195112 & 1443.73 \\
\hline UF9 & 0.088857 & 0.188603 & 0.160832 & 0.047975 & 0.218993 & 1270.73 \\
\hline UF10 & 0.473865 & 0.744428 & 0.781509 & 0.134987 & 1.043141 & 1359.30 \\
\hline
\end{tabular}

within 30 times independent execution for dealing with each problem. The Figure 8 is of the NSGA-II [13] produced in its best run among 30 independent runs for CEC'09 test instances [57]. The PFs displayed by enhanced AMALGAM are more promising than NSGA-II [13] in terms of diversity and proximity.

We have also plotted 30 PFs altogether estimated by enhanced AMALGAM in the figure 5 and the figure 9 exhibited by NSGA-II [13] in all 30times independent runs over the problems UF1-UF4 and UF7-UF10. These figures clearly indicate that enhanced AMALGAM has tackled the most CEC'09 test instances more effectively and spend less CPU time dealing with each test problem as compared to NSGA-II [13].

The problems UF5 and UF6 have not been tackled by both algorithms as per demand and genetic drift has been occurred in their respective population due to the presence of highly multi-modality in these problems like UF5-UF6. The search process of both algorithms are get stuck in the local basin of attraction of these problems and due to this both algorithms have not shown further improvement dealing with these problems.

\section{CONCLUSION AND FURTHER WORK}

Different operators suite different optimization and search problems. The dynamic use of multiple operators in the EA framework has exhibited good performance on complicated MOPs, [31], [39], [34], [36], [32], [41], [40]. A multialgorithm genetically adaptive multi-objective (AMALGAM) has recently been developed for solving both single objective [53] and multi-objective optimization problems [52]. In this paper, we have suggested new adaptive resource allocation procedure and developed a enhanced version of AMALGAM to cope with CEC'09, [57], and ZDT test problems [53]. The suggested algorithm has shown promising results on most test problems compared to NSGA-II [13] in terms of proximity and diversity. Furthermore, the suggested algorithm is more efficient which is desirable when solving real-word problems where time can be an issue. In the future, we intend to examine the performance of enhanced AMALGAM over real-world problems such as:

- Tubular permanent magnet linear synchronous motor 

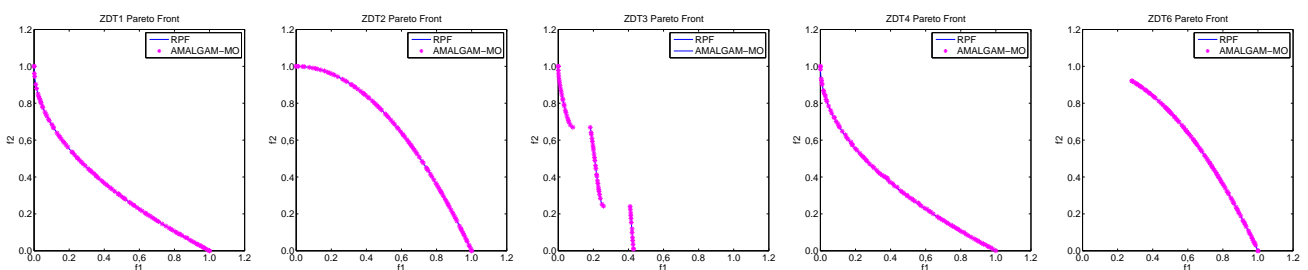

Fig. 2. Plots of the final non-dominated solutions in the objective space displayed by enhanced AMALGAM in its best run among 30 independent runs over ZDT1-ZDT4 and ZDT6 problems.
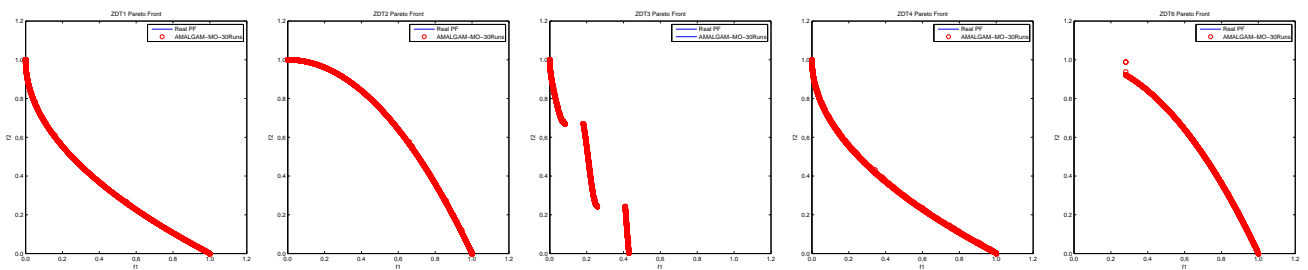

Fig. 3. Plots of the $30 \mathrm{PFs}$ altogether in the objective space displayed by enhanced AMALGAM for ZDT1-ZDT4 and ZD6 problems.
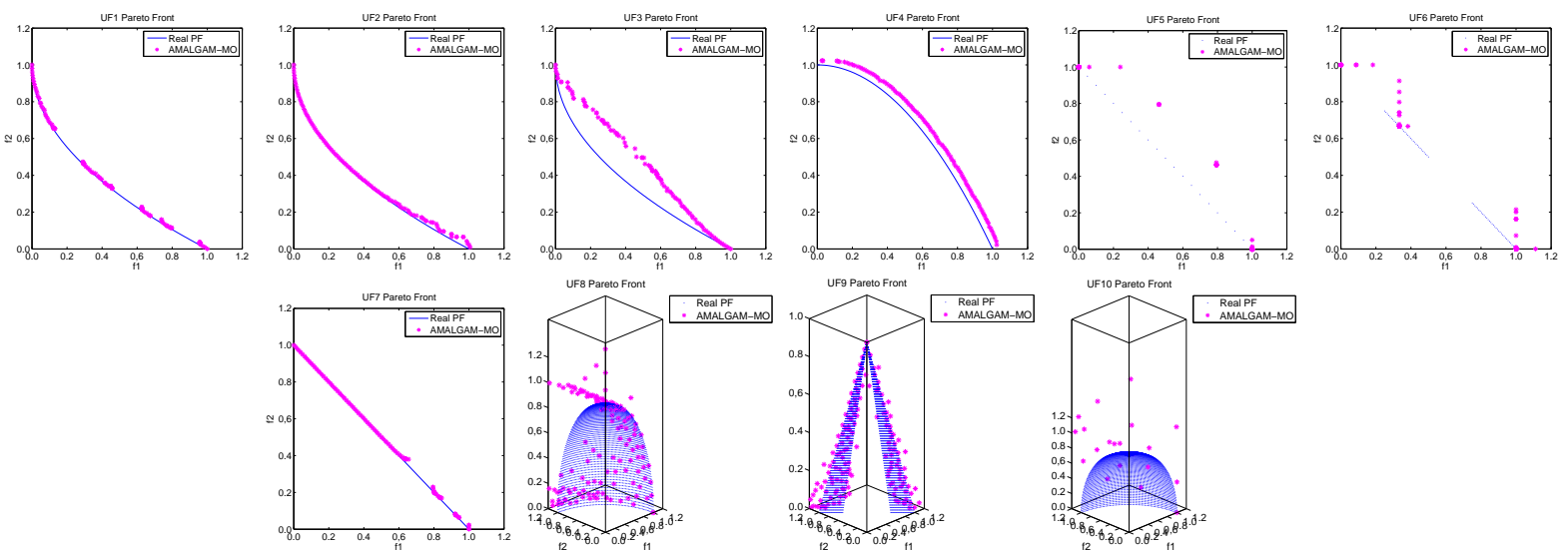

Fig. 4. Plots of the final non-dominated solutions in the objective space displayed by enhanced AMALGAM in its best run among 30 independent runs over UF1-UF10 problems.
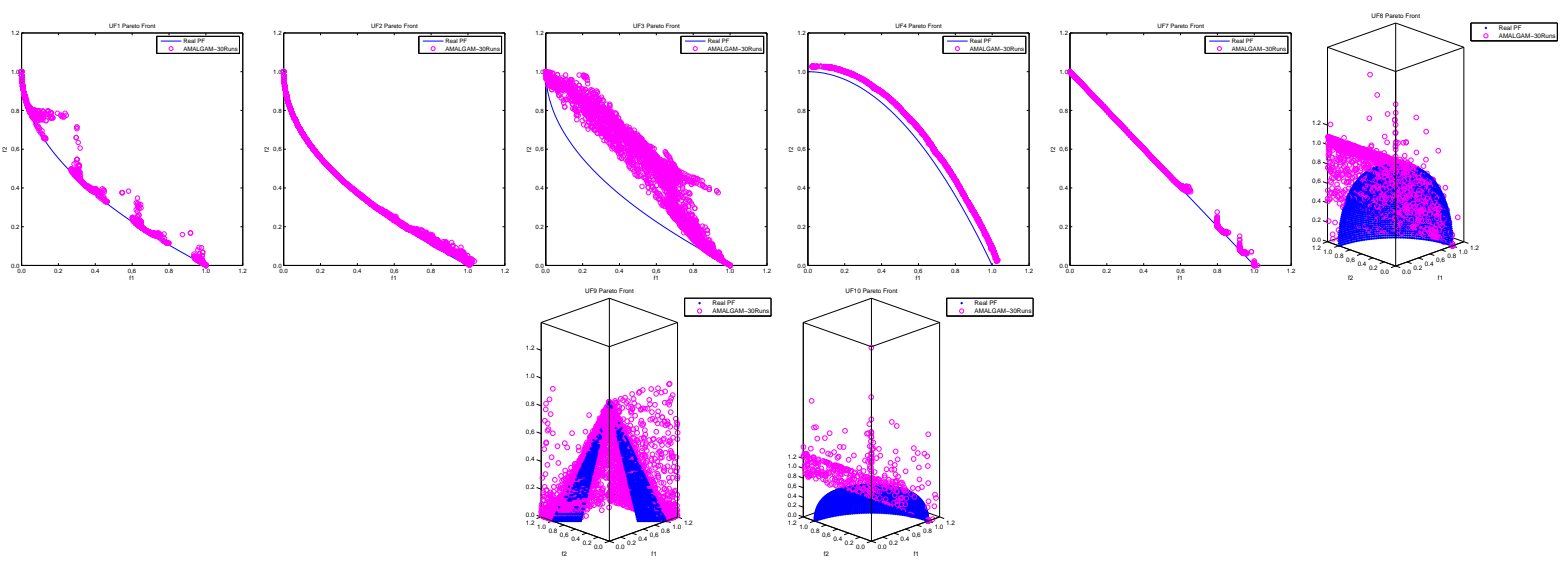

Fig. 5. Plots of the 30 PFs altogether in the objective space displayed by enhanced AMALGAM for UF1-UF4 and UF7-UF10 problems. 

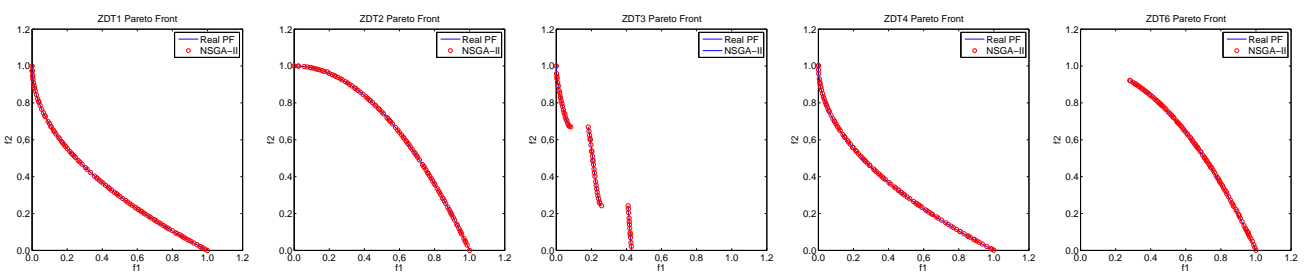

Fig. 6. Plots of the final non-dominated solutions in the objective space displayed by NSGA-II [13] in its best run among 30 independent runs over ZDT1-ZDT4 and ZDT6 problems.
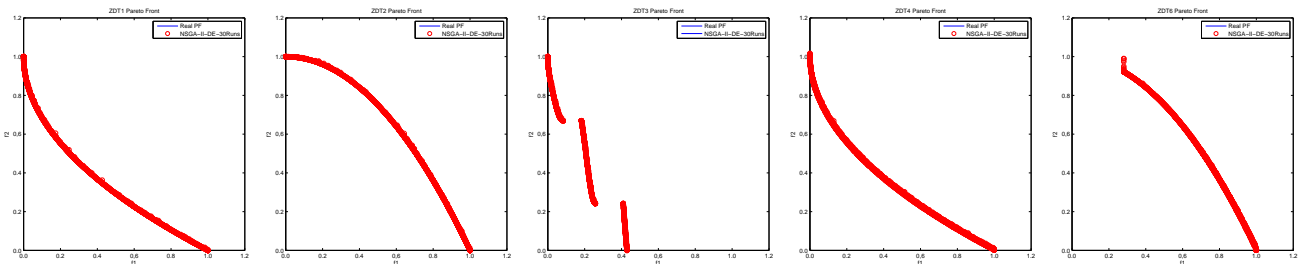

Fig. 7. Plots of the $30 \mathrm{PFs}$ altogether in the objective space produced by NSGA-II [13] for ZDT1-ZD4 and ZDT6 problems.
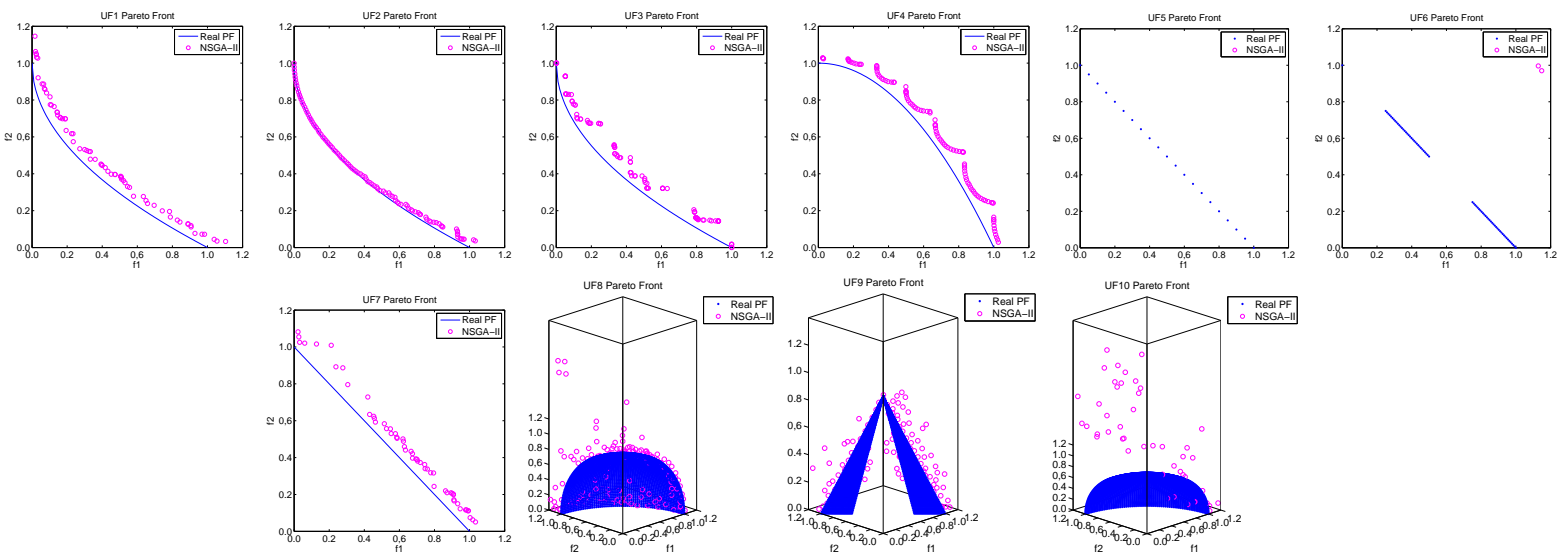

Fig. 8. Plots of the final non-dominated solutions in the objective space display by NSGA-II [13] in its best run among 30 independent runs over UF1-UF10 problems.
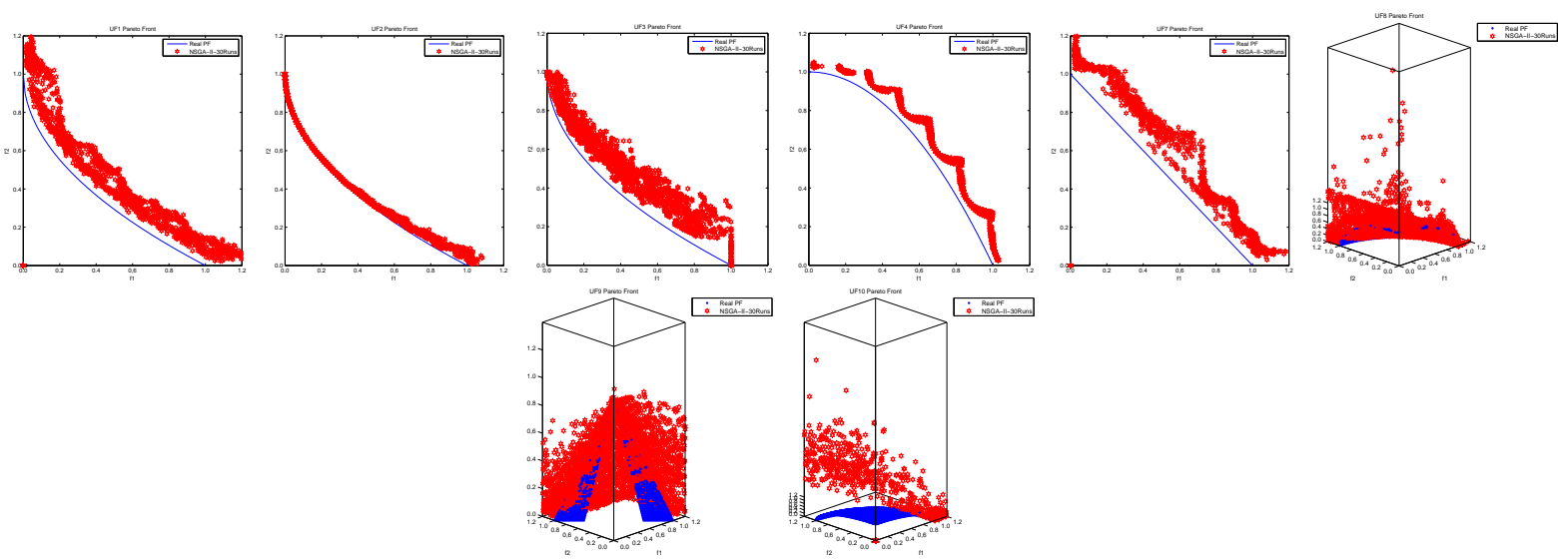

Fig. 9. Plots of the 30 PFs altogether in the objective space display by NSGA-II [13] for UF1-UF4 and UF7-UF10 problems. 
(TPMLSM).

- Cancer chemotherapy problems.

- Fuzzy multi-objective reliability redundancy allocation problem.

- Multiobjective Engineering design problems.

- Multi-Objective Capacitated Arc Routing Problem.

- Passive Vehicle Suspension Optimization.

- Multi-objective mobile agent-based Sensor Network Routing.

- Oil and Gas Well Drilling problems.

We will also examine the effect of various enhanced versions of differential evolution [38] with self-adaptive capabilities in our proposed algorithm on the problems mentioned above as well as other complicated test problems.

\section{REFERENCES}

[1] N. Al Moubayed, A. Petrovski, and J. McCall, "A novel smart multiobjective particle swarm optimisation using decomposition," in Proceedings of the 11th international conference on Parallel problem solving from nature: Part II, ser. PPSN'10. Berlin, Heidelberg: Springer-Verlag, 2010, pp. 1-10.

[2] A. Auger, J. Bader, D. Brockhoff, and E. Zitzler, "Hypervolumebased multiobjective optimization: Theoretical foundations and Practical Implications," Theoretical Computer Science, vol. 425, pp. 75-103, 2012.

[3] J. Bader, "Hypervolume-Based Search for Multiobjective Optimization: Theory and Methods," Ph.D. dissertation, ETH Zurich, Switzerland, 2010.

[4] J. Bader and E. Zitzler, "HypE: An Algorithm for Fast HypervolumeBased Many-Objective Optimization," Evolutionary Computation, vol. 19, no. 1, pp. 45-76, 2011.

[5] N. Beume, B. Naujoks, and M. Emmerich, "SMS-EMOA: Multiobjective Selection based on Dominated hypervolume," European Journal of Operational Research, vol. 181, no. 3, pp. 1653-1669, 2007.

[6] Carlos and R. L. Becerra, "Evolutionary Multi-Objective Optimization in Materials Science and Engineering," Materials and Manufacturing Processes, vol. 24, no. 2, pp. 119-129, 2009.

[7] P. C. Chang, S. H. Chen, Q. Zhang, and J. L. Lin, "MOEA/D for Flowshop Scheduling Problems," in IEEE Congress on Evolutionary Computation, CEC'08, june 2008, pp. 1433-1438.

[8] C.-M. Chen, Y.-P. Chen, and Q. Zhang, "Enhancing MOEA/D with Guided Mutation and Priority Update for Multi-Objective Optimization," in Proceedings of the IEEE Congress on Evolutionary Computation, CEC 2009, Trondheim, Norway, 18-21 May, 2009, 2009, pp. 209-216.

[9] C. A. C. Coello, "A Comprehensive Survey of Evolutionary-Based Multiobjective Optimization Techniques," Knowledge and Information Systems, vol. 1, pp. 269-308, 1999.

[10] C. A. C. Coello, G. B. Lamont, and D. A. V. Veldhuizen, Evolutionary Algorithms for Solving Multi-Objective Problems (Genetic and Evolutionary Computation). Secaucus, NJ, USA: Springer-Verlag New York, Inc., 2006.

[11] C. A. Coello Coello, G. B.Lamont, and D. A. Veldhuizen, Evolutionary Algorithms for Solving Multi-Objective Problems. Kluwer Academic Publishers, New York,, March 2002.

[12] K. Deb, Multi-Objective Optimization Using Evolutionary Algorithms, 2nd ed., S. Ross and R. Weber, Eds. John Wiley and Sons Ltd, 2002.

[13] K. Deb, A. Pratap, S. Agarwal, and T.Meyarivan, "A Fast and Elitist Multiobjective Genetic Algorithm:NSGA-II," IEEE Transsation On Evolutionary Computation, vol. 6, no. 2, pp. 182-197, 2002.

[14] D.H.Phan and J.Suzuki, "R2-IBEA: R2 indicator based evolutionary algorithm for multiobjective optimization," in 2013 IEEE Congress on Evolutionary Computation (CEC), June 2013, pp. 1836-1845.
[15] R. Eberhart and J.Kennedy, "A New Optimizer Using Particle Swarm Theory," in Proceedings of the Sixth International Symposium on Micro Machine and Human Science, MHS'95, oct. 1995, pp. 39-43.

[16] E.Zitzler and L. Thiele, "Multiobjective Evolutionary Algorithms: A Comparative Case Study and the Strength Pareto Evolutionary Algorithm," IEEE Transactions on Evolutionary Computation, vol. 3, no. 4, pp. 257-271, 1999.

[17] C. Fonseca and P. Fleming, "Genetic Algorithms for Multiobjective Optimization: Formulation, Discussion and Generalization," in Proceedings of the 5th International Conference on Genetic Algorithms. San Francisco, CA, USA: Morgan Kaufmann Publishers Inc., 1993, pp. 416423.

[18] —, "An Overview of Evolutionary Algorithm in Multi-Objective Optimization," Evolutionary Computation, vol. 3, no. 1, pp. 1-16, 1995.

[19] J. Horn, N. Nafpliotis, and D. E. Goldberg., "A Niched Pareto Genetic Algorithm for Multiobjective Optimization," in Proceedings of the First IEEE Conference on Evolutionary Computation, CEC'94, 1994.

[20] H. Ishibuchi and T. Murata, "Multi-Objective Genetic Local Search Algorithm and Its Application to Flowshop Scheduling," IEEE Transactions on Systems, Man and Cybernetics, vol. 28, no. 3, pp. 392-403, 1998.

[21] H. Ishibuchi and T. Murata., "Multi-Objective Genetic Local Search Algorithm." in Proceedings of the Third IEEE International Conference on Evolutionary Computation, I. T. Fukuda and T. Furuhashi, Eds., Nagoya, Japan, 1996, pp. 119-124.

[22] H. Ishibuchi, N. Tsukamoto, Y. Sakane, and Y. Nojima, "Indicatorbased Evolutionary Algorithm with Hypervolume Approximation by Achievement Scalarizing Functions," in Proceedings of the 12th Annual Conference on Genetic and Evolutionary Computation, ser. GECCO '10. New York, NY, USA: ACM, 2010, pp. 527-534.

[23] J.D.Knowles and D.W.Corne, "Local Search, Multiobjective Optimization and the Pareto Archived Evolution Strategy," in Proceedings of the Third Australia-Japan Joint Workshop on Intelligent and Evolutionary Systems, Ashikaga Institute of Technology, Japan, November 1999, pp. 209-216.

[24] K.Deb and R. Agrawal, "Simulated Binary Crossover for Continuous Search Space," Complex System, vol. 9, pp. 115-148, 1995.

[25] W. Khan, "Hybrid multiobjective evolutionary algorithm based on decomposition," PhD, Department of Mathematical Sciences, University of Essex, Wivenhoe Park, CO4 3SQ, Colchester, UK, January 2012.

[26] W. Khan and Q. Zhang, "MOEA/D-DRA with Two Crossover Operators," in UK Workshop on Computational Intelligence (UKCI), September 2010, pp. 1-6.

[27] J. Knowles and D. Corne, "The Pareto Archived Evolution Strategy: A new Baseline Algorithm for Pareto Multiobjective Optimization," in Proceedings of the IEEE Congress on Evolutionary Computation (CEC', 99), Piscatay, NJ, JULY 1999, pp. 98-105.

[28] A. Konak, D. W.Coit, and A. E. Smith, "Multi-Objective Optimization Using Genetic Algorithms:A tutorial," Reliability Engineering and System Safety, vol. 91, no. 7, p. 9921007, July 2006.

[29] M. Koppen, "On the benchmarking of multiobjective optimization algorithm." in Knowledge-Based Intelligent Information and Engineering Systems, Proceedings Lecture Notes in Artificial Intelligence, 2773, 2003, pp. 379-385.

[30] H. Li and Q. Zhang, "Multiobjective Optimization Problems With Complicated Pareto Sets: MOEA/D and NSGA-II," IEEE Transsation On Evolutionary Computation, vol. 13, no. 2, pp. 284-302, April 2009.

[31] W. K. Mashwani, "A Multimethod Search Approach Based on Adaptive Generations Level," in Seventh International Conference on Natural Computation(ICNC'11), Shanghai, China, 26-28 July, 2011, pp. 2327.

[32] _ _ "Hybrid Multiobjective Evolutionary Algorithms: A Survey of the State-of-the-art," International Journal of Computer Science Issues, vol. 8, no. 6, pp. 374-392, 2011.

[33] — , "Integration of NSGA-II and MOEA/D in Multimethod Search Approach: Algorithms," in Proceedings of the 13th Annual Conference Companion on Genetic and Evolutionary Computation. ACM, 2011, pp. $75-76$.

[34] - "MOEA/D with DE and PSO: MOEA/D-DE+PSO," in The Thirty-first SGAI International Conference on Innovative Techniques 
and Applications of Artificial Intelligence, Cambridge, UK, December, 2011, pp. 217-221.

[35] — - "Comparison of Evolutionary Algorithm over Multiobjective Optimization Problems," in Proceeding of International Conference on Modeling and Simulation (ICOMS), Air University Islamabad, Pakistan, 2013.

[36] _ " "Comprehensive Survey of the Hybrid Evolutionary Algorithms," International Journal of Applied Evolutionary Computation (IJAEC), vol. 4, no. 2, pp. 1-19, July 2013.

[37] — _Performanance of AMALGAM over CEC'09 Test Instances," in Proceeding Third International Conference on Aerospace Science and Engineering (ICASE'13), 2013.

[38] _ "Enhanced versions of Differential Evolution: state-of-the-art survey," International Journal Computing Sciences and Mathematics, vol. 5, no. 2, pp. 107-126, 2014.

[39] W. K. Mashwani and A. Salhi, "A Decomposition-Based Hybrid Multiobjective Evolutionary Algorithm with Dynamic Resource Allocation," Applied Soft Computing, vol. 12, no. 9, pp. 2765-2780, 2012.

[40] _ " "Multiobjective Evolutionary Algorithm Based on Multimethod with Dynamic Resources Allocation," Applied Soft Computing, vol. 39, pp. 292-309, 2016

[41] W. K. Mashwani, A. Salhi, M. A. Jan, R.A.Khanum, and M. Sulaiman, "Impact Analysis of Crossovers in Multiobjective Evolutionary Algorithm," Science International Journal, vol. 27, no. 6, pp. 4943-4956, December 2015.

[42] W. K. Mashwani and A. Salhi, "Multiobjective Memetic Algorithm Based on Decomposition," Applied Soft Computing, vol. 21, pp. 221243, 2014.

[43] K. M. Miettinien, Nonlinear Multiobjective Optimization, ser. Kluwer's International Series. Norwell, MA: Academic Publishers Kluwer, 1999.

[44] N.Srinivas and K.Deb, "A Multiobjective Optimization using Nondominated Sorting in Genetic Algorithms," J Evol Comput, vol. 2, no. 3, pp. 221-248, 1994.

[45] P.Moscato, "On evolution, Search, Optimization, Genetic Algorithms and martial arts: Towards memetic algorithms," California Institute of Technology, Pasadena, California, USA, Tech. Rep. Caltech Concurrent Computation Program 826, 1989.

[46] R.Storn and K.V.Price, "Differential Evolution - A Simple and Efficient Heuristic for Global Optimization over Continuous Spaces," ICSI, Technical Report TR-95-012, 1995.

[47] _ - "Differential Evolution - a Simple and Efficient Heuristic for Global Optimization over Continuous Spaces," J.Global Opt, vol. 11, no. 4, pp. 341-359, December 1997.

[48] J. D. Schaffer, "Multiple Objective Optimization with Vector Evaluated Genetic Algorithms," in Proceedings of the 1st International Conference on Genetic Algorithms (ICGA), Pittsburgh, USA, July. Lawrence Erlbaum Associates, 1985, pp. 93-100.

[49] D. Simon, Evolutionary Optimization Algorithms: Biologically Inspired and Population-Based Approches to Computer Intelligence. John Wiley \& Sons, 2013.

[50] S. Tsutsui, M. Yamamura, and T. Higuchi, "Multi-Parent Recombination with Simplex Crossover in Real coded Genetic Algorithms," in Proceeding of GECCO-99, 1999, pp. 657-374.

[51] D. A. V. Veldhuizen, "Multiobjective Evolutionary Algorithms: Classifications, Analyses, and New Innovations," Graduate School of Engineering of the Air Force Institute of Technology Air University, Tech. Rep., 1999.

[52] J. A. Vrugt and B. A. Robinson, "Improved Evolutionary Optimization from Genetically Adaptive Mutimethod Search," Proceedings of the National Academy of Sciences of the United States of America: PNAS (USA), vol. 104, no. 3, pp. 708-701, 16th Jaunuary 2007.

[53] J. A. Vrugt, B. A. Robinson, and J. M. Hyman, "Self-Adaptive Multimethod Search for Global Optimization in Real-Parameter Spaces," IEEE Transsation On Evolutionary Computation, vol. 13, no. 2, pp. 243-259, April 2009.

[54] Q. Zhang and H. Li, "MOEA/D: A Multiobjective Evolutionary Algorithm Based on Decomposition " IEEE transaction on Evolutionary Computation, vol. 11, no. 6, pp. 712-731, December 2007.
[55] Q. Zhang, H. Li, D. Maringer, and E. P. K. Tsang, "MOEA/D with NBI-style Tchebycheff Approach for Portfolio Management," in Proceedings of the IEEE Congress on Evolutionary Computation, CEC 2010, Barcelona, Spain, 18-23 July 2010, 2010, pp. 1-8.

[56] Q. Zhang, W. Liu, and H. Li, "The Performance of a New Version of MOEA/D on CEC'09 Unconstrained MOP Test Instances," IEEE Congress On Evolutionary Computation (IEEE CEC 2009), Trondheim, Norway, pp. 203-208, May, 18-21 2009.

[57] Q. Zhang, A. Zhou, S. Zhaoy, P. N. Suganthany, W. Liu, and S. Tiwariz, "Multiobjective Optimization Test Instances for the CEC 2009 Special Session and Competition," Technical Report CES-487, 2009.

[58] S.-Z. Zhao, P. N. Suganthan, and Q. Zhang, "Decomposition-Based Multiobjective Evolutionary Algorithm With an Ensemble of Neighborhood Sizes," IEEE Trans. Evolutionary Computation, vol. 16, no. 3, pp. 442-446, 2012.

[59] A. Zhou, B.-Y. Qu, H. Li, S.-Z. Zhao, P. N. Suganthan, and Q. Zhang., "Multiobjective evolutionary algorithms: A survey of the state-of-theart," Swarm and Evolutionary Computation, vol. 1, pp. 32-49, 16 March 2011, online publised.

[60] E. Zitzler, M. Laumanns, and L. Thiele, "SPEA2: Improving the Strength Pareto Evolutionary Algorithm," Computer Engineering and Networks Laboratory (TIK), ETH Zurich, Zurich, Switzerland, TIK Report 103, 2001

[61] E. Zitzler and L. Thiele, "An Evolutionary Approach for Multiobjective Optimization: The Strength Pareto Approach," Computer Engineering and Networks Laboratory (TIK), ETH Zurich, TIK Report 43, May 1998.

[62] E. Zitzler, K. Deb, and L. Thiele, "Comparsion of Multiobjective Evolutionary Algorithms: Emperical Results," Evolutionary Computation, vol. 8, no. 2, pp. 173-195, 2000.

[63] E. Zitzler and S. Knzli, "Indicator-Based Selection in Multiobjective Search," in Parallel Problem Solving from Nature - PPSN VIII, ser. Lecture Notes in Computer Science, X. Yao, E. Burke, J. Lozano, J. Smith, J. Merelo-Guervs, J. Bullinaria, J. Rowe, P. Tino, A. Kabn, and H.-P. Schwefel, Eds. Springer Berlin Heidelberg, 2004, vol. 3242, pp. $832-842$.

[64] E. Zitzler, L. Thiele, M. Laumanns, C. M. Fonseca, and V. G. da Fonseca, "Performance Assessment of Multiobjective Optimizers: An Analysis and Review," IEEE Transactions on Evolutionary Computation, vol. 7, pp. 117-132, 2003 